\title{
Comparison between the tie-over and Karydakis procedure in chronic sacrococcygeal pilonidal sinus
}

\author{
Mohammad Moazeni Bistgani ${ }^{\mathbb{1}}$ \\ 'Department of Surgery, Faculty of Medicine, Shahrekord University of Medical Sciences Shahrekord, Iran
}

*Corresponding Author: Mohammad Moazeni Bistgani, Department of Surgery, Faculty of Medicine, Shahrekord University of Medical Sciences, Shahrekord, Iran. Tel: +989131812974, Fax: +983832224492, E-mail: dr_m_moazeni@yahoo.com

\begin{abstract}
Background and aims: Pilonidal sinus in the sacrococcygeal region is a disease with high postoperative morbidity and discomfort for the patient. Although there are various therapeutic modalities to manage these patients, there is still controversy about choosing the best surgical technique to decrease the recurrence rate, patient discomfort, and duration of disability after surgery. The present study aimed to compare the results obtained from two surgery methods including tie-over procedure (TOP) and Karydakis procedure (KP) for the treatment of sacrococcygeal pilonidal sinus.

Methods: In this single-blind clinical trial, 70 patients with the diagnosis of pilonidal sinus disease were divided into two equal groups $(\mathrm{n}=35$ each) and treated randomly with surgical excision and TOP or KP by the same surgeon in the Kashani educational hospital in Shahrekord, Iran from 2009 and 2011. The patients were followed up until December 2019.

Results: According to the results, after excision of sacrococcygeal pilonidal sinus, the primary closure of the wound by tie-over sutures had better results than KP in order of pain sore during the first month after surgery $(P<0.001)$, duration of disability $(P<0.001)$, wound repair time $(P<0.001)$, and patient satisfaction $(P=0.019)$.

Conclusion: Our results showed TOP was a better procedure compared to KP for the treatment of chronic pilonidal sinus due to higher acceptance results in patients undergoing surgery.

Keywords: Sacrococcygeal pilonidal sinus, Suture techniques, Karydakis procedure, Tie-over procedure, Wound healing
\end{abstract}

Received: 23 May 2020, Accepted: 12 August 2020, ePublished: 30 June 2021

\section{Introduction}

Pilonidal sinus disease usually affects young adults and is three to four times more common in men than women (1); its incidence is reported as 26 in 100000 people (2). In recent studies, acquired factors were known responsible for the etiopathogenesis of the disease (1). Although the common site of disease is the sacrococcygeal region, some reports in the umbilicus (3), breast (4), intermammary (5), axillary (6), penis, vulvae (7), and nasal bridge (8) have also been reported with unignorable potentially incidentally finding of malignancy in the lesion (9). History and physical examination are usually sufficient for diagnosis, although the role of sonography in the better detection of lesion was reported in some studies (10). Currently, acute abscesses are incised and drained, and chronic cases are usually treated by surgical excision followed by primary closure (11). Although several modalities or aimed treatm ents (such as laser, endoscopy, phenol application) (1214) have been advocated, it seems that the best result is obtained from surgical excision and primary surgical wound closure. However, the technique used is still a very controversial concept (1-15).

Numerous surgical techniques include Bascom procedure, Karydakis procedure (KP), excision, leaving the wound to granulate, excision and primary closure with midline, asymmetric incisions or excision, and closure using local flaps, which include Rhomboid and Limberg flaps (16). In the literature, Z-plasty (17) and V-Y flaps (18) have been described as the treatment options. Such a diversity in treatment options suggests that no single technique has been considered as the best choice. In this regard, patient satisfaction includes the following: postoperative ambulation with low intensity of pain, low postoperative complications, diminished hospital stay, and in some cases cosmetic results.

This study aimed to compare results achieved in patients with sacrococcygeal pilonidal sinus treated with surgical excision and primary surgical wound closure with either tie-over procedure (TOP) or KP.

\section{Materials and Methods}

This single-blind clinical trial included 70 patients with sacrococcygeal pilonidal sinus. After institutional review board approval (identifier: IRCT201301073156N2; https:// www.irct.ir/trial/3207) and obtaining a written informed consent from all patients, the author performed all procedures at Kashani educational hospital in Shahrekord, Iran. Information for the study was obtained from the case notes by the first author. Out of a total of 73 diagnosed cases, three patients who were in acute conditions or had 
a recurrent sacrococcygeal pilonidal sinus were excluded from the study. Patients were randomly allocated to undergo surgery with TOP or KP in two groups $(n=35$ each). We recorded age, sex, weight, height, body mass index (BMI), smoking, hemoglobin ( $\mathrm{Hb})$, family history, and duration of reported symptoms in the questionnaire.

The gluteal region, including the area surrounding the intergluteal sulcus was shaved 30 minutes before surgery. The operation was performed with the patient in a prone position and all procedures were performed under spinal anesthesia by the same surgeon. All patients received a single intravenous dose of 1-gram Keflin half an hour before surgery.

\section{Tie-over procedure}

Using this technique, after complete excision of the pilonidal sinus with symmetric ellipsoid incision by scalpel down to the pre-sacral fascia and hemostasis with diathermy, the surgical field was reassessed to ensure any remained tract and hairs (Figure 1). Then, flaps were mobilized from the two sides. The edge of the wound was touched by atraumatic instruments gently to prevent wound micro-laceration (thereby diminished woundsrelated complications) (Figure 2, 3). After passing of fullthickness skin and subcutaneous fat and pre-sacral fascia with $1 / 0$ prolene thread from two edges in the three or four separate sutures, skin, and subcutaneous fat between them, the approximated and wounds edges repaired with $2 / 0$ prolene, also in separated simple vertical mattress suture. The operation completed without leaving dead spaces and no need to drain. Then, the repaired area was covered by two or three arranged formal surgical nonopaque gauzes and tied previous tension sutures over the gauzes and dressing applied on the surgical area (Figure $4)$.

\section{Karydakis procedure}

In the KP group, a standard asymmetric ellipsoid incision was created and all the sinus tracts were removed completely down to the presacral fascia. The flap was mobilized to achieve natal cleft lateralization. Before placing the subcutaneous sutures, an 18-French suction drain was placed in the resultant dead space and brought

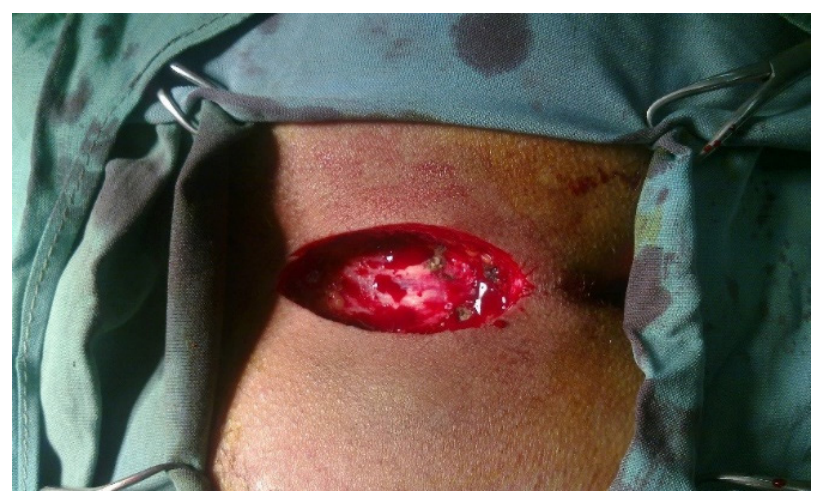

Figure 1. Complete excision of pilonidal sinus (prone position).

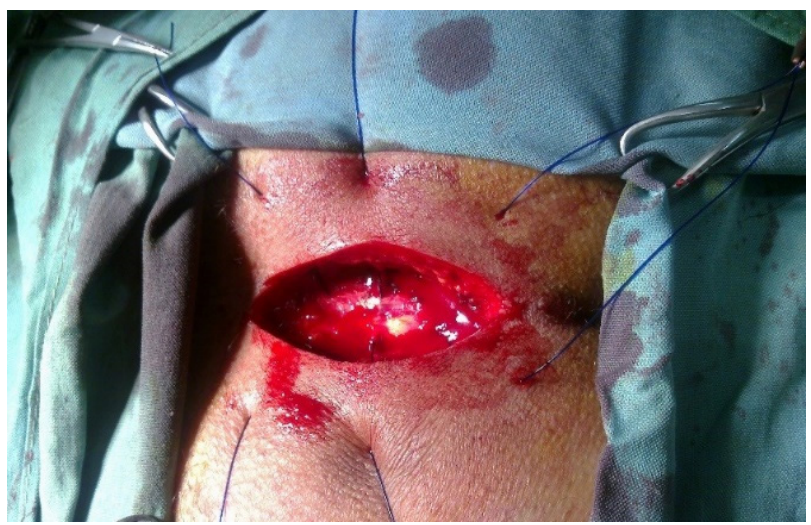

Figure 2. 1/0 prolene suture passed from two edges of the wounds (including presacral fascia) to prevent dead space and minimize wound tension and decrease wound dehiscence.

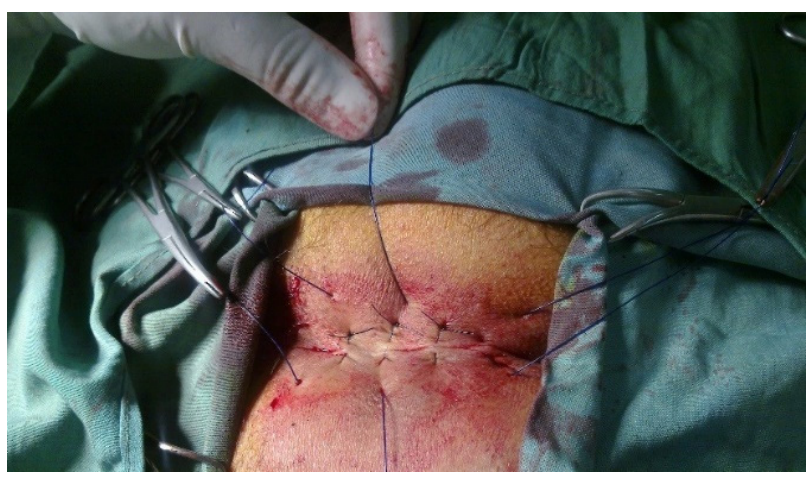

Figure 3. So-called tension sutures, tied over, but yarns preserved and not cut off for farther covering of the wounds with gauzes.

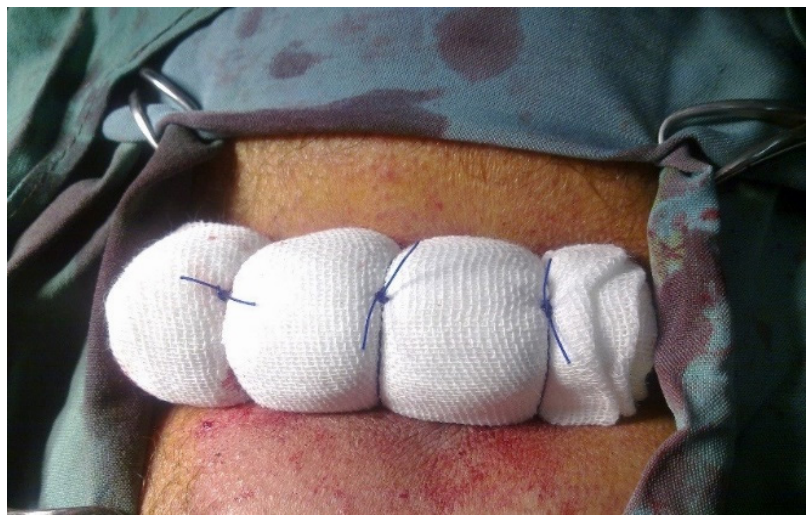

Figure 4. Tie-over technique by tying previous tension sutures over the gauzes.

out well laterally through a separate stab incision. The subcutaneous tissue was closed in two to three layers using 2-0 vicryl sutures. The skin was closed with 4-0 polypropylene using a vertical mattress suture and a dressing applied over the surgical area.

\section{Postoperative follow-up}

During their 24-hour hospital stay, patients were given intravascular injections of $25 \mathrm{mg}$ of pethidine on-demand and Keflin $1 \mathrm{~g}$ QID. All patients received oral cephalexin $500 \mathrm{mg}$ QID and ibuprofen $400 \mathrm{mg}$ TID until 1-week post- 
operation. Regardless of the method used, without gauzes under tie-over, daily dressings are advised in any method. On discharge date, patients were recommended to pay attention to hygiene rules and neither sit nor use a semisitting position for two weeks; walking was not restricted. Taking a shower was restricted in TOP patients for one week and in KT patients for three days. Also, patients were shown how to estimate the daily fluid drainage from the suction drain in KP or wound in TOP. The suction drain in $\mathrm{KP}$ was removed when the effluent was less than $20 \mathrm{~mL}$ per 24 hours for two consecutive days. Finally, patients were asked to remove their gluteal hair as necessary for six months after the operation. In the TOP group, tieover suture was removed one week after the operation, and if the wound repaired, two weeks after the operation. In the KP group, the suture was removed two weeks after the operation if the wound repaired. The patients were allowed to return to the work after 10 days of the surgery. After discharge, operative time and duration of hospital stay were recorded. Patients were followed up by physical examination weekly for the first four weeks after surgery and at the end of third month. A visual analog scale (VAS; $0=$ no pain and $10=$ very severe [unbearable] pain) was used to measure postoperative pain. The pain was evaluated on the morning of the first day after surgery, before discharge, and weekly until four weeks, then the mean of pain VAS was recorded. Postoperative complications were recorded with emphasis on wound infection, wound dehiscence, and fluid collections under the flap. Time to return to daily activities and duration of disability was determined and recorded by asking patients about when they were able to do their daily activities and return to work. Wound repair time was determined and recorded by the surgeon. Patient satisfaction was evaluated and recorded three months after operation using a fivepoint Likert scale ( $1=$ total unsatisfied; $2=$ unsatisfied; $3=$ neutral; $4=$ satisfied; and $5=$ very satisfied). A checklist including telephone number and address was developed to record the latest results of patients. A recurrent disease was reported if observed at any time throughout the study period and the end of the study.

Results

Data analysis was performed using SPSS software version 18. The student t-test was used to compare groups regarding continuous variables, and the Fisher's exact test was used for categorical variables. $P<0.05$ was considered as statistically significant. Demographic parameters of all patients and their results are illustrated in Table 1.

The age range of our patients was 15-53 years (mean age: $26.9 \pm 7.6)$ without significant difference in the two groups $(P<0.05)$.

There were 56 males (80\%) and 14 females (20\%) without a significant difference in the two groups $(P<0.05)$. The male-to-female ratio of the pilonidal sinus disease was $4: 1$.

No significant differences were observed between the groups regarding weight, height, BMI, smoking, positive family history, $\mathrm{Hb}$, and duration of complaint.

There was no significant difference between the groups regarding the mean operative time $(P=0.903)$. Wound repair time and the duration of disability were significantly shorter in TOP patients compared to KP ones $(P<0.001)$. The mean pain score was significantly lower in TOP patients $(P<0.001)$ (Table 2$)$.

After three months from operation, the mean satisfaction score was significantly higher in the TOP group than in the KT group $(P<0.019)$

One patient in the TOP group had full-thickness wound dehiscence; this complication occurred in three patients in the KP group but there was no significant difference between the groups $(P=0.614)$. This condition was managed with daily dressings and eventually healed completely without adverse sequelae.

No patient in the KP group had postoperative wound infection, but this complication occurred in one patient in the TOP group. Regarding wound infections, there was no significant difference between the groups $(P=1)$; the wounds were managed with antibiotics along with daily dressings, which did not lead to wound breakdown. Two patients in the TOP group had fluid collection under the

Table 1. Demographics variables of the patients in the TOP and KP groups

\begin{tabular}{|c|c|c|c|c|}
\hline Variables & & $\begin{array}{c}\text { TOP } \\
\text { Mean } \pm \text { SD/No. }(\%)\end{array}$ & $\begin{array}{c}\text { KP } \\
\text { Mean } \pm \text { SD/No. }(\%)\end{array}$ & $P$ value \\
\hline Number & & 35 & 35 & - \\
\hline \multirow{2}{*}{ Sex } & Male & $29(82.9 \%)$ & $27(77.1 \%)$ & \multirow{2}{*}{0.55} \\
\hline & Female & $6(17.1 \%)$ & $8(22.9 \%)$ & \\
\hline Mean age (y) & & $28.1 \pm 7.3$ & $25.7 \pm 8$ & 0.189 \\
\hline Weight (kg) & & $76.4 \pm 12.1$ & $75.8 \pm 13$ & 0.85 \\
\hline Height (cm) & & $173.1 \pm 9.7$ & $173.5 \pm 8.6$ & 0.86 \\
\hline BMI & & $25.5 \pm 4.2$ & $25 \pm 2.8$ & 0.61 \\
\hline Smoking & & $18(51.4 \%)$ & $10(28.6 \%)$ & 0.051 \\
\hline Positive family history & & $13(37.1 \%)$ & $17(48.6 \%)$ & 0.334 \\
\hline $\mathrm{Hb}(\mathrm{mg} / \mathrm{dL})$ & & $14.3 \pm 1.7$ & $14.8 \pm 1.5$ & 0.201 \\
\hline Duration of complaint (month) & & $10.8 \pm 12.9$ & $12.9 \pm 15.4$ & 0.539 \\
\hline
\end{tabular}


Table 2. Distribution of Results

\begin{tabular}{lccc}
\hline Variables & TOP & KP & Mean \pm SD/No. (\%) \\
\hline Mean operative time (min) & $14.8 \pm 4.3$ & $14.2 \pm 3.1$ & 0.903 \\
Mean pain score (VAS) & $1.9 \pm 1$ & $5 \pm 2.1$ & $<0.001$ \\
Mean patient satisfaction (LS) & $7.8 \pm 1.8$ & $6.7 \pm 2$ & 0.019 \\
Wound repair time (day) & $15.6 \pm 3.3$ & $22.1 \pm 8$ & $<0.001$ \\
Duration of disability (day) & $12.9 \pm 3.8$ & $19.7 \pm 11.3$ & $<0.001$ \\
Postoperative infection(yes) & $1(2.9 \%)$ & 0 & $1^{*}$ \\
Fluid collections under the flap (yes) & $2(5.7 \%)$ & $3(8.6 \%)$ & $1^{*}$ \\
Wound dehiscence (yes) & $1(2.9 \%)$ & $3(8.6 \%)$ & 0.614 \\
Recurrence (yes) & 0 & $1(2.9 \%)$ & $1^{*}$ \\
\hline S. likert scale & & &
\end{tabular}

LS: likert scale

flap; this complication occurred in three patients in the $\mathrm{KP}$ group, though there was no significant difference between the groups $(P=1)$. This complication in KP was attributed to the drain occlusion that was managed with drain washing, but in TOP group it was managed with repeated aspiration.

After a follow-up time of 9-10 years, no patient in the TOP group had a postoperative recurrence of disease, but this complication occurred in one patient in the KP group; however, there was no significant difference between the groups $(P=1)$.

\section{Discussion}

The best technique for the treatment of sacrococcygeal pilonidal sinus is still controversial. In line with trying to solve some dilemmas in this issue, especially the achievement of better patient's condition, there usually continues low morbidity after surgery. The present singleblind clinical trial compared the results of two techniques of TOP and the KP in the surgical management of chronic sacrococcygeal pilonidal sinus disease.

In our study, sex, mean age, weight, height, BMI, smoking, positive family history, $\mathrm{Hb}$, and duration of complaint did not have a significant difference between the two groups. The age and sex distributions of our patients were consistent with those reported in the literature (1).

At first glance, TOP technique is a simple addition of secondary reinforcement tie; according to the literature, the TOP technique has better results than simple Mattresssuture. TOP technique is conventionally used as a dressing technique of some difficult handled parts of the body such as shoulder, groin traumatic wounds and burn (19), interdigital web wounds; it is also used for coverage of skin grafts (20-22).

In the present study, we did not find significant differences between the groups regarding operation time and short- and long-term complications; however, there was a significant difference between the groups in wound repair time, mean pain score, patient satisfaction, and duration of disability.

Lower mean pain score in the TOP group may be attributed to the absence of a requirement for suction drain and pillow effects of Tie-over gauzes. Although cosmetic issues have less importance in developing countries compared to developed countries, we found that patients in TOP group had higher levels of satisfaction in this regard ( $8 \pm 1.8$ vs. $6.7 \pm 2, P=0.019$ ).

In this study, TOP patients had less duration of postoperation disability than KP patients $(12.9 \pm 3.8$ vs. $19.7 \pm$ 11.3 days, $P<0.001$ ); this might be due to the fact that some pillow modes affect part of Tie-Over gauzes in buttock area, which allows the patient to be more comfortable for early mobilization and walking (especially 2 or 3 days after the operation).

Many studies compared simple primary closure techniques with closure by various flaps methods in terms of reducing wound infections; these techniques have been associated with different rates of wound site infection. While wound site infection rates were between 1.93$11.45 \%$ after primary closure technique (23-24), it was 1 vs 0 in TOP and KP groups, indicating no significant difference $(P=1)$.

This result may be due to using pre- and post-operative antibiotics in two groups, but it emphasizes the importance of avoiding surgery in the presence of active sepsis and the need for aggressive preoperative eradication of infection before attempting flap surgery for sacrococcygeal pilonidal sinus disease.

The formation of post-operative under flap collection is mostly related to technique, surgical wounds dead space, delayed ambulation (25), and increased risk of this early complication. The main cause of delayed ambulation is a pain, especially in patients with excisional surgery in the lower portion of the trunk (such as pilonidal sinus surgery).

On the very first days after the recovery from anesthesia, pillow effects of Tie-over gauzes in TOP can be helpful for the patients for not suffering from pain in movement, and thereby forming less collection under flap.

The routine use of suction drains has been previously advocated by Bessa (26) for preventing postoperative under flap fluid collections; in TOP, we did not use the suction drain. Lower under flap collection incidence in TOP patients may be attributed to pillow effects of Tie- 
over gauzes in preventing surgical wounds dead space, early ambulation, and less painful movement. Willingness and readiness in wound care caused no drainage in TOP patients.

In previous studies, the wound dehiscence rates were reported from $0 \%$ to $6 \%$ for the classic KP $(27,28)$; in our study, this rate was $8.6 \%$ for KP and $2.9 \%$ for TOP. Although the rate of this complication was not significantly different between the two groups, the pillow effects of Tie-over gauzes in TOP can be helpful for the patients to lessen the risk of wound dehiscence.

In the study by Stauffer et al, 89583 operated patients for pilonidal sinus with the Karydakis \& Bascom approaches were analyzed; the recurrence was $0.2 \%$ after 12 months and $0.6 \%$ after 24 months of surgery. Primary midline closure exhibited long-term recurrence up to $67.9 \%$ after 20 years of surgery (29).

In our study, after a follow-up time of 9-10 years, no patient in the TOP group had a postoperative recurrence of disease, but this complication occurred in one patient in the KP group. Our follow-up period was relatively long, but recurrence rates were low and similar in the two groups.

Yardimci compared the outcomes of the KT and a combination of pit excision and sinus tract ablation using a $1470 \mathrm{~nm}$ diode laser (the pit excision (PE) + laser ablation technique (LAT)). After a mean follow-up time of 25 months; recurrence rates were $1(3.6 \%)$ in the KT group and $1(3.3 \%)$ in the PE + LAT group (30).

\section{Conclusion}

It was shown that pillow effect of gauzes applied to the wounds can help the patients for their earliest incision wound healing. In the same way, pressure over the incision with the least pain decreased the probability of other early postoperative complications. Although both techniques are safe for the treatment of pilonidal sinus disease, the TOP is better than the KT.

\section{Conflict of Interests}

The authors declare no conflict of interests.

\section{Ethical Approval}

This article has been extracted from a research project approved by Shahrekord University of Medical Sciences, Iran (code: IR.SKUMS.REC.91-3-13).

\section{Funding/Support}

This study was funded by Shahrekord University of Medical Sciences.

\section{Acknowledgments}

The authors would like to thank the Surgery Department of Shahrekord University of Medical Sciences for the sincere collaboration and the staff of Kashani University Hospital for assisting in data collection (Grant no. 1128).

\section{References}

1. Sahsamanis G, Samaras S, Mitsopoulos G, Deverakis T,
Dimitrakopoulos G, Pinialidis D. Semi-closed surgical technique for treatment of pilonidal sinus disease. Ann Med Surg (Lond). 2017;15:47-51. doi: 10.1016/j. amsu.2017.02.004.

2. Dessily M, Charara F, Ralea S, Allé JL. Pilonidal sinus destruction with a radial laser probe: technique and first Belgian experience. Acta Chir Belg. 2017;117(3):164-8. doi: 10.1080/00015458.2016.1272285.

3. Kaplan M, Kaplan ET, Kaplan T, Kaplan FC. Umbilical pilonidal sinus, an underestimated and little-known clinical entity: report of two cases. Am J Case Rep. 2017;18:267-70. doi: 10.12659/ajcr.903016.

4. Lahiri R, Mullen R, Ashton MA, Abbott NC, Pollock AM. Pilonidal abscess in the breast: a case report. J Surg Case Rep. 2014;2014(6):rju061. doi: 10.1093/jscr/rju061.

5. Sunkara A, Wagh D, Harode S. Intermammary pilonidal sinus. Int J Trichology. 2010;2(2):116-8. doi: 10.4103/09747753.77526.

6. Sengul I, Sengul D, Mocan G. Axillary pilonidal sinus: a case report. N Am J Med Sci. 2009;1(6):316-8.

7. Kushwaha P, Merritt A, Aslam MB. A rare case of vulval pilonidal sinus: incidental diagnosis. BMJ Case Rep. 2015;2015. doi: 10.1136/bcr-2014-204693.

8. Junaid M, Ahmed SQ, Kazi M, Ali NS. Pilonidal sinus involving the nasal bridge: a rare manifestation. BMJ Case Rep. 2015;2015. doi: 10.1136/bcr-2015-209948.

9. Eryılmaz R, Bilecik T, Okan I, Ozkan OV, Coşkun A, Sahin M. Recurrent squamous cell carcinoma arising in a neglected pilonidal sinus: report of a case and literature review. Int J Clin Exp Med. 2014;7(2):446-50.

10. Mandava A, Ravuri PR, Konathan R. High-resolution ultrasound imaging of cutaneous lesions. Indian J Radiol Imaging. 2013;23(3):269-77. doi: 10.4103/09713026.120272.

11. Rashidian N, Vahedian-Ardakani J, Baghai-Wadji M, Keramati MR, Saraee A, Ansari K, et al. How to repair the surgical defect after excision of sacrococcygeal pilonidal sinus: a dilemma. J Wound Care. 2014;23(12):630-3. doi: 10.12968/jowc.2014.23.12.630.

12. Dragoni F, Moretti S, Cannarozzo G, Campolmi P. Treatment of recurrent pilonidal cysts with nd-YAG laser: report of our experience. J Dermatolog Treat. 2018;29(1):65-7. doi: 10.1080/09546634.2017.1329513.

13. Giarratano G, Toscana C, Shalaby M, Buonomo O, Petrella G, Sileri P. Endoscopic pilonidal sinus treatment: longterm results of a prospective series. JSLS. 2017;21(3). doi: 10.4293/jsls.2017.00043.

14. Emiroğlu M, Karaali C, Esin H, Akpınar G, Aydın C. Treatment of pilonidal disease by phenol application. Turk J Surg. 2017;33(1):5-9. doi: 10.5152/ucd.2016.3532.

15. Gecim IE, Goktug UU, Celasin H. Endoscopic Pilonidal Sinus Treatment Combined With Crystalized Phenol Application May Prevent Recurrence. Dis Colon Rectum. 2017;60(4):405-407. doi: 10.1097/DCR.0000000000000778.

16. Bali İ, Aziret M, Sözen S, Emir S, Erdem H, Çetinkünar S, et al. Effectiveness of Limberg and Karydakis flap in recurrent pilonidal sinus disease. Clinics (Sao Paulo). 2015;70(5):3505. doi: 10.6061/clinics/2015(05)08.

17. Rao J, Deora H, Mandia R. A retrospective study of 40 cases of pilonidal sinus with excision of tract and Z-plasty as treatment of choice for both primary and recurrent cases. Indian J Surg. 2015;77(Suppl 2):691-3. doi: 10.1007/s12262- 
013-0983-4.

18. Demiryilmaz I, Yilmaz I, Peker K, Celebi F, Cimen O, Isik A, et al. Application of fasciocutaneous V-Y advancement flap in primary and recurrent sacrococcygeal pilonidal sinus disease. Med Sci Monit. 2014;20:1263-6. doi: 10.12659/ msm. 890752.

19. Patil RK, Koul AR. Modified way of applying a tie-over dressing. Indian J Plast Surg. 2016;49(1):124-6. doi: 10.4103/0970-0358.182251

20. Joyce KM, Joyce CW, Mahon N, Kelly JL. Use of a barbed suture tie-over technique for skin graft dressings: a case series. Arch Plast Surg. 2015;42(3):341-5. doi: 10.5999/ aps.2015.42.3.341.

21. Pulvermacker B, Chaouat M, Seroussi D, Mimoun M. Tieover dressings in full-thickness skin grafts. Dermatol Surg. 2008;34(1):40-3. doi: 10.1111/j.1524-4725.2007.34006.x.

22. Joyce KM, Joyce CW, Mahon N, Kelly JL. Use of a barbed suture tie-over technique for skin graft dressings: a case series. Arch Plast Surg. 2015;42(3):341-5. doi: 10.5999/ aps.2015.42.3.341.

23. Khan PS, Hayat H, Hayat G. Limberg flap versus primary closure in the treatment of primary sacrococcygeal pilonidal disease; a randomized clinical trial. Indian J Surg. 2013;75(3):192-4. doi: 10.1007/s12262-012-0430-y.

24. Enshaei A, Motearefi S. Comparison of two surgical methods, primary closure and rotational flap, in patients with chronic pilonidal sinus. Glob J Health Sci. 2014;6(7 Spec No):18-22. doi: 10.5539/gjhs.v6n7p18.

25. Srivastava V, Basu S, Shukla VK. Seroma formation after breast cancer surgery: what we have learned in the last two decades. J Breast Cancer. 2012;15(4):373-80. doi: 10.4048/ jbc.2012.15.4.373.

26. Bessa SS. Comparison of short-term results between the modified Karydakis flap and the modified Limberg flap in the management of pilonidal sinus disease: a randomized controlled study. Dis Colon Rectum. 2013;56(4):491-8. doi: 10.1097/DCR.0b013e31828006f7.

27. Ates M, Dirican A, Sarac M, Aslan A, Colak C. Short and long-term results of the Karydakis flap versus the Limberg flap for treating pilonidal sinus disease: a prospective randomized study. Am J Surg. 2011;202(5):568-73. doi: 10.1016/j.amjsurg.2010.10.021.

28. Ersoy E, Devay AO, Aktimur R, Doganay B, Ozdoğan M, Gündoğdu RH. Comparison of the short-term results after Limberg and Karydakis procedures for pilonidal disease: randomized prospective analysis of 100 patients. Colorectal Dis. 2009;11(7):705-10. doi: 10.1111/j.14631318.2008.01646.x.

29. Stauffer VK, Luedi MM, Kauf P, Schmid M, Diekmann $\mathrm{M}$, Wieferich $\mathrm{K}$, et al. Common surgical procedures in pilonidal sinus disease: a meta-analysis, merged data analysis, and comprehensive study on recurrence. Sci Rep. 2018;8(1):3058. doi: 10.1038/s41598-018-20143-4.

30. Yardimci VH. Outcomes of two treatments for uncomplicated pilonidal sinus disease: Karydakis flap procedure and sinus tract ablation procedure using a $1,470 \mathrm{~nm}$ diode laser combined with pit excision. Lasers Surg Med. 2020;52(9):848-54. doi: 10.1002/lsm.23224. 